\title{
Analysis of the Signal over Noise Ratio of the hodoscope determined by Monte Carlo calculation
}

\author{
J. Di Salvo*,****, S. Mirotta**, V. Chevalier*** \\ ****Corresponding author: jacques.disalvo@irsn.fr
}

\begin{abstract}
The CABRI experimental pulse reactor, located at the Cadarache nuclear research center, southern France, is devoted to the study of Reactivity Initiated Accidents (RIA) for the purpose of the CABRI International Program (CIP), managed by IRSN in the framework of an OECD/NEA agreement. The hodoscope equipment installed in the CABRI reactor is an almost unique online fuel motion monitoring system, thanks to the measurement of the fast neutrons emitted during a power pulse by a tested rod positioned inside a dedicated test loop reproducing PWR conditions. This system is dedicated to the analysis of fuel displacement. Hence, one of the most important parameter measured by the hodoscope detectors is the Signal over Noise Ratio (SNR), characterizing the fraction of neutrons directly coming from the test rod ("signal") over neutrons coming from the core ("noise").
\end{abstract}

It is interesting to calculate the SNR in order to define some quantitative criterions to improve hodoscope measurements and to understand if any modification linked to the test loop may significantly change this essential parameter.

Another parameter of interest is the so-called "scattering coefficient", which corresponds to the fraction of neutrons coming from the test rod and being scattered between their birth and their detection. This parameter is used to enhance the analysis of the fuel displacement which may happen during the power transient.

In this article, the method used to calculate the SNR using MCNP6.2 Monte Carlo code will be detailed. Because the hodoscope detectors are located far away from the test rod (up to 4 meters), a 2D model of CABRI core and instrumentation has been implemented. No variance reduction techniques have been used to solve this problem in order to record the place of birth of neutron which contributes to the different scores with the goal to perform a detailed analysis of the SNR. This strategy allows to access numerically to the "scattering coefficient".

Finally, the comparison between calculated and measured SNR for a case study will be carried out. A quite good agreement between the 2D simulations and experiments recently performed in the CABRI reactor has been obtained.

Index Terms - CABRI, Hodoscope, MCNP, RIA, Neutron transport.

*J. Di Salvo Author, was with IRSN/PSN-RES/SEREX/L2EP, Cadarache, Saint Paul Lez Durance 13115 FRANCE (email: jacques.disalvo@ irsn.fr), **S. Mirotta Author, was with IRSN/PSN-RES/SEREX/L2EP, Cadarache, Saint Paul Lez Durance 13115 FRANCE (email: salvatore.mirotta@irsn.fr), ***V. Chevalier Author, was with IRSN/PSN-RES/SEREX/ L2EP, Cadarache, Saint Paul Lez Durance 13115 FRANCE (email: vincent.chevalier@irsn.fr)

\section{INTRODUCTION}

Gor enhancing safety of nuclear power plants (NPP), the French Nuclear Safety and Radiation Protection Institute (IRSN) carries out some experimental programs in order to improve the understanding of the fuel behavior under accident conditions. One of them is the CABRI International Program, managed and funded by IRSN under OECD/NEA umbrella, devoted to study of Reactivity Initiated Accidents (RIA) in representative PWR conditions [1].

For this purpose, the renovation of the CABRI facility has been conducted by CEA, its operator. This pool type reactor is designed to submit to a RIA a test rod placed into the center of the core.

The power transients are generated by the unique reactivity injection system of CABRI [2]. 96 tubes (so-called "transient rods", visible in Figure 1) located in 4 banks among the CABRI fuel rods are pressurized with ${ }^{3} \mathrm{He}$ gas. The very fast depressurization of this strong neutron absorber into a discharge tank, through two flow channels (low and high flow rates), is then finely adjusted to trigger the desired power pulse.

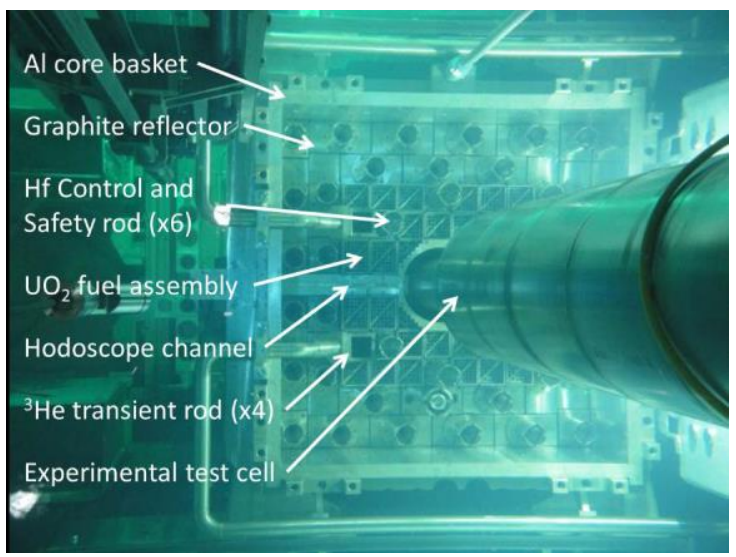

Figure 1 -View of the CABRI reactor from the top

The CABRI facility is equipped with two nondestructive measurement systems operated by IRSN:

- The IRIS facility, for performing X-ray radiography and tomography imaging before and after a power transient thanks to a linear electron accelerator, as well as quantitative gamma scanning analyses; 
- The Hodoscope, an online fuel motion measurement system, which aims at analyzing the fuel motion deduced from the detection of fast neutrons emitted by the tested rod, in real time (with a time step of $1 \mathrm{~ms}$ ) during the transient [3].

This article present the numerical model for the evaluation of the Signal over noise ratio of the hodoscope measurement system which is a fundamental parameter to observe the fuel displacement (if any) during the power excursion. More in details, in section II of this article, a brief description of the meaning of the signal over noise ratio will be given. In section III the 2D model developed with MCNP6 [4] will be discussed. Furthermore, in section IV results and a comparison to experimental values will be analyzed. Finally, current studies will be presented, and conclusion is given in section V. Some definitions are reminded in section VI.

\section{SignAl OVER NOISE RATIO DEFINITION}

The hodoscope measurement system, installed on the CABRI reactor, is used to detect and measure online, during power transients, fuel displacement which may occur on the test rod. The hodoscope system is made by a huge collimator about $3 \mathrm{~m}$ long, which is placed in front of the test rod $1 \mathrm{~m}$ far and allows to filter in space and energy both, neutrons coming from the core and those coming from the test rod. The collimator has 153 lines of sight arranged in a matrix of 51 rows and 3 columns in order to assure both axial and radial discretization of the test rod as well as the possibility to use the signal coming from one of these columns to normalize the signal during power excursion. Beyond each line of sight of the collimator two different detectors are installed. The first is a ${ }^{237} \mathrm{~Np}$ fission chamber (energy threshold cross section at $600 \mathrm{keV}$ ) and the second one is proton recoils detectors (energy threshold set by electronic system at $400 \mathrm{keV}$ ) [5].

In this context the observable used to detect fuel displacement during power transient and therefore the signal over noise ratio (SNR) are fast neutrons coming from the test rod [6].

Only these neutrons are useful for our purpose, all other neutrons (thermal neutrons, fast neutrons produced in CABRI rods, etc...) do not contain any useful information for fuel displacement evaluation. Hence, for this reason, they contribute to the "noise" on the measurement. With both, detector's technology and collimator geometry it is possible to measure only fast neutrons coming from the test rod (which contribute to the useful "signal").

The main difficulty in the measurement of the SNR is due to the small amount of these neutrons coming from the test rod compared to all other neutrons present in the reactor.

A specific methodology is used to evaluate this parameter by the means of a Monte-Carlo simulation.

In Figure 2, a schematic cross section of the CABRI reactor and the line of sight of the hodoscope collimator are represented. In this figure it is possible to see that if the center column of the hodoscope collimator is placed in front of the test rod, detectors placed beyond the center column aim the test rod and therefore capture neutrons coming from it.

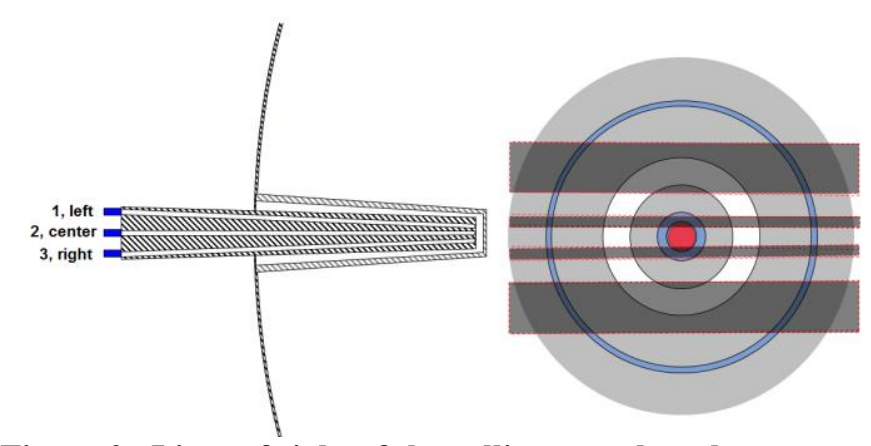

Figure 2-Lines of sight of the collimator when the center column aims entirely the test rod

The system of Equation 1 for the detectors counting rate can be written when the collimator is placed in its centered position. The left column detector as well as the right column detector can measure fast neutrons coming from the reactor (n) and those coming from the test rod scattered into the structural materials of the reactor $(\alpha s)$. Only the center column can measure the fast neutrons coming from the test rod (s), in complement with the noise and the scattered neutrons.

$\left\{\begin{array}{l|l|}c_{l}=n+\alpha s & \\ c_{c}=n+\alpha s+s & \text { Equation } 1 \\ c_{r}=n+\alpha s & \end{array}\right.$

Using Equation 1, Equation 2 can be written as follow.

$$
R=\frac{c_{c}}{\frac{c_{l}+c_{r}}{2}}=\frac{s}{n+\alpha s}+1=\frac{s}{N}+1 \quad \text { Equation 2 }
$$

The signal over noise ratio can be measured by the ratio between the counting rates of the hodoscope detectors. It is important to note that it is not possible to measure directly the signal over pure noise ratio $\mathrm{s} / \mathrm{n}$, because the scattering coefficient has to be determined firstly.

\section{NUMERICAL MODEL}

\section{A. Geometry description}

The CABRI reactor model was built using MCNP6.2. In Figure 3, a radial cross section of the reactor at $\mathrm{z}=0$ plane is shown. It is a $2 \mathrm{D}$ model which does not take into account the axial control rod position nor the axial power flux. This simplification was needed because of the poor statistic obtained with the 3D model in the vicinity of the hodoscope detectors. In fact, detectors are located very far from the reactor and a non-analog simulation did not give useful results since the place of birth of the neutron is lost. More precisely, following simplifications were implemented in the 2D model:

- Axial grids were not modeled;

- Control and security rods were completely inserted into the reactor;

- The Helium 3 pressure into the transient rod was adjusted in order to keep the system critic;

- The overall water pool was not modeled. 


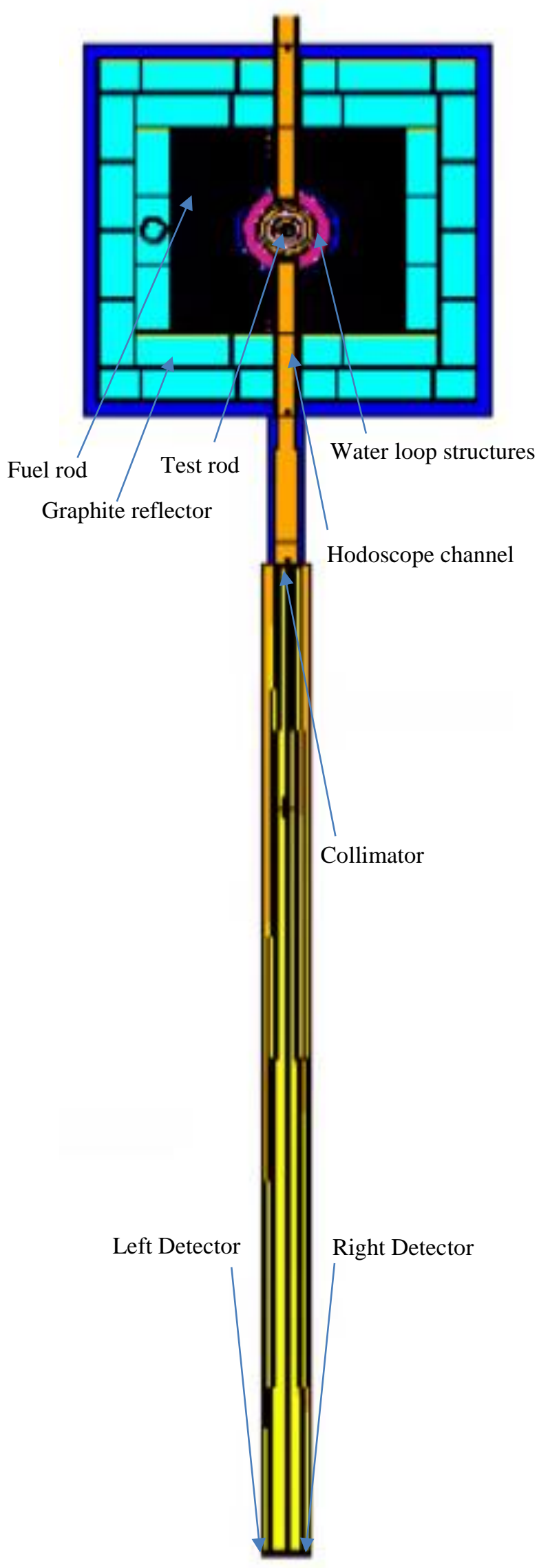

Figure 3 - Radial cross section of CABRI reactor
Once the CABRI model implemented, two different test rods were put in place in the center of the reactor: a fresh $\mathrm{UO}_{2}$ enriched up to $4.25 \%$ in ${ }^{235} \mathrm{U}$, and an aluminum one.

Moreover, several fictive control volumes were placed in the line of sight of the center hodoscope channel in order to observe the attenuation of the neutrons current through the collimator

\section{B. Tally description}

Tallies are used in MCNP6 to obtain an estimation of a physical observables into the model. For this study F4 tally was considered. F4 tallies were split in two energy bins:

- The first one collects all neutrons with energy below $600 \mathrm{keV}$;

- The second group takes into account neutrons with energy above $600 \mathrm{keV}$.

In this article fast fluxes which will be enounced, correspond to all neutrons with an energy above $600 \mathrm{keV}$ which is the threshold value for ${ }^{237} \mathrm{~Np}$ fission cross section. In the simulation, we collect the neutrons above $600 \mathrm{keV}$ coming from both the core and the test rod into the three detectors region. Scoring the fast fluxes all over three detectors region is enough to estimate the SNR. It important to observe that the scattering coefficient is not available using this method.

Hence, another option of the MCNP6 code was used to evaluate the proportion of the neutrons produced into the test rod and those scattered into the water loop structures.

For this evaluation, the knowledge of the birthplace of each neutron contributing to the tally results is required. To do that, FU tally tagging option was activated and the tally results were split into several contributions.

Two different ways to treat elastic scattering contribution can be used within this option:

- $\quad$ scattered particles lose their birthplace tag and they are putted into elastic scattered bin (option TAG 1);

- $\quad$ scattered particles still keep the birthplace tag, elastic scatter bin is empty in this case (option TAG 3).

Hence, for TAG 1 calculation, four different bins were defined for this study:

1. Bin 1 , it takes into account source neutrons born by fission into the test rod (the signal $\mathrm{s}$, red line in Figure 4);

2. Bin 2, which takes into account $(\mathrm{n}, \mathrm{xn})$ or $\left(\mathrm{n}, \mathrm{n}^{\prime}\right)$ neutrons produced into the test rod by other neutrons (they are also part of the signal s, black dash-dotted line in Figure 4);

3. Bin 3, takes into account all scattered neutrons independently from where they were born (which contains the scattered part of the signals as - blue dashed line in Figure 4 -, a part of the signal s itself black dashed line in Figure 4 -, and all the neutrons coming from everywhere in the core and being scattered once time in their history);

4. Bin 4 takes into account all other neutrons which come from the reactor (not represented in the Figure 4 ), and (n,n') reactions of neutrons coming from the test rod (grey dash-dotted line in Figure 4); 
In the case of TAG3, the filling logic for those bins is quite different: the $3^{\text {rd }}$ bin is always empty because all scattered neutron keep the birthplace tag and they are placed into bin 1 , bin 2 or bin 4 .

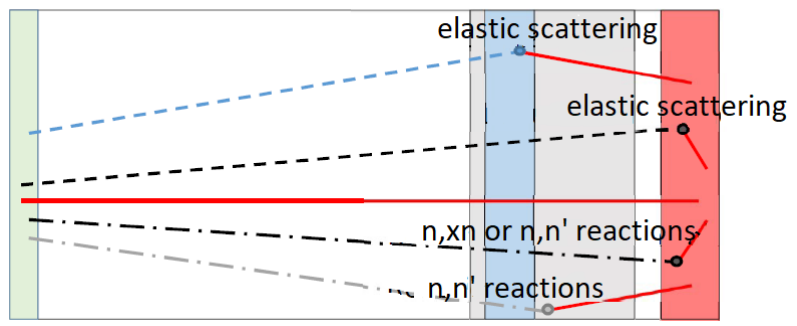

Test Rod

Pressurized water loop structures

Water

Detection volume

Figure 4 - All different neutron reactions produced by neutrons coming from the test rod (axial cut)

Therefore, for TAG3 calculation, Equation 3 can be written. The sum of the contributions of bin 1 and bin 2 takes into account all neutrons produced into the test rod.

\begin{tabular}{|c|l|}
\hline$s+\alpha s=b_{1_{c, T A G 3}}+b_{2_{c, T A G 3}}$ & Equation 3 \\
\hline
\end{tabular}

For TAG1 calculation, Equation 4 can be written.

$$
\begin{array}{l|l}
s=b_{1_{c, T A G 1}}+b_{2_{c, T A G 1}}+\varphi_{e l, c} & \text { Equation } 4
\end{array}
$$

This equation takes into account the term $\varphi_{e l, c}$, which corresponds to the fraction of neutrons produced into the test rod and elastically scattered in itself, participating to the signal in addition with bin 1 and bin 2 .

The evaluation of this fraction was possible using the neighboring channels using Equation 5, because they don't aim the test rod so that the signal is not contained in the counting rate of these channels.

$$
\begin{array}{|c|l|}
\hline \varphi_{e l, c}=b_{3_{c, T A G 1}}-\frac{b_{3_{r, T A G 1}}+b_{3_{l, T A G 1}}}{2} & \text { Equation 5 } \\
\hline
\end{array}
$$

Finally it is possible to write Equation 6 to obtain the scatter coefficient for the CABRI reactor.

$$
\alpha=\frac{b_{1_{c, T A G 3}}+b_{2_{c, T A G 3}}}{b_{1_{c, T A G 1}}+b_{2_{c, T A G 1}}+\varphi_{e l, c}}-1 \quad \text { Equation } 6
$$

In conclusion, with TAG 3 option it is possible to split the contribution to the tally which comes from the core (noise $n$ ) and the contribution to the tally due to the test rod (signal $s+\alpha s$ ) within a slight approximation due to neutrons coming from the test rod and scattered by inelastic collisions from the water loop structures. Hence, with TAG 1 option it is possible to split the contribution of $\mathrm{s}$ from that due to neutrons coming from the test rod and elastically scattered into the water loop structures $(\alpha s)$.

\section{RESUltS}

Results are presented in this section with uncertainties evaluated at $2 \sigma$, which means a confidence interval of $95 \%$. Three different results will be presented in this section:

- The collimator importance for the increasing of SNR;

- The evaluation of the SNR for two different test rods;

- The preliminary evaluation of the scattering coefficient.

\section{A. Collimator role}

The importance of the hodoscope collimator is shown in this section. In Figure 5 the radial distribution of the fast flux $(>600 \mathrm{keV})$ is presented. Two different simulations were performed, the first one without the collimator and the second one with the collimator. In the first case, without the collimator the flux into the detectors zone is reduced by a factor 100. With the collimator, the reduction factor is more important: the neutrons flux is reduced by a factor of 500 . Comparing the two calculations, the flux loss is quite important, but the interesting result is that the Signal over Noise ratio is equal to $11,4 \% \pm 0,4 \%$ without the collimator and reaches $40,2 \% \pm 1,1 \%$ with the collimator. This means that the collimator is crucial for a good SNR evaluation, complementary to the axial discretization.

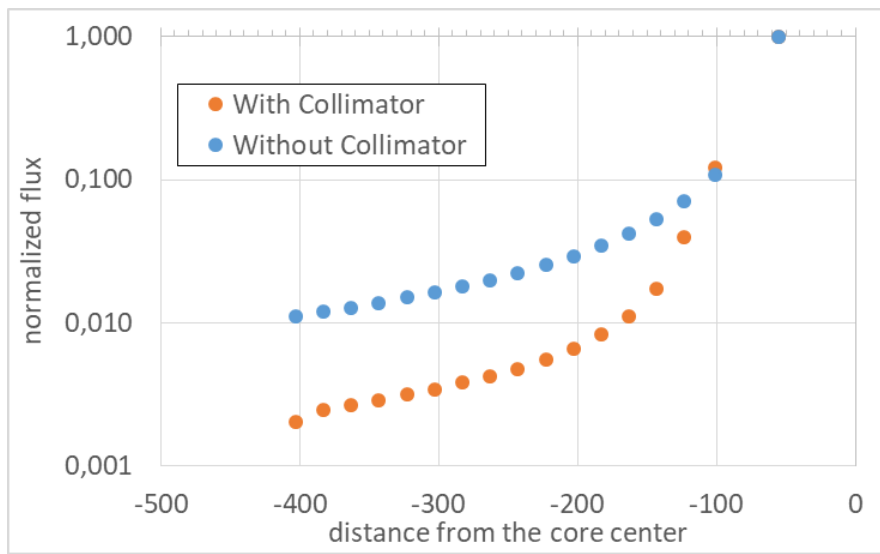

Figure 5 - Fast neutron flux with and without the hodoscope collimator

\section{B. SNR evaluation}

Thanks to the methodology presented in II, it is possible to evaluate the SNR ratio.

Table 1 shows results obtained for fresh $\mathrm{UO}_{2}$ test rod for the three detectors beyond the collimator.

Table 1 - Tally score for the three channels for fresh $\mathrm{UO}_{2}$ test rod loaded into the experimental cell

\begin{tabular}{|l|l|}
\hline & Flux (a.u.) \\
\hline Neutron Flux for right channel $c_{\mathrm{r}}$ & $4.1410^{-08} \pm 1.4 \%$ \\
\hline Neutron Flux for center channel $\mathrm{c}_{\mathrm{c}}$ & $5.3310^{-08} \pm 1.2 \%$ \\
\hline Neutron Flux for left channel $\mathrm{c}_{1}$ & $4.1710^{-08} \pm 1.4 \%$ \\
\hline
\end{tabular}

$\mathrm{S} / \mathrm{N}_{\mathrm{UO} 2}=\mathbf{2 8 . 3 \%} \pm \mathbf{1 . 7 \%}$ 
The SNR is contained in the interval [26.5\%; 30.2\%] for fresh $\mathrm{UO}_{2}$ test rod. The measured value obtained during a reactor power plateau, by using the same technique is $27.0 \%$ $\pm 2.0 \%$. Comparison of calculated result versus experimental one in the case of the fresh $\mathrm{UO}_{2}$ fuel are quite in good agreement.

The second case study concerns the simulated signal over noise ratio for a non-fissile material. In this theoretical case the value should be 0 , because no neutrons (or at least very few) come from the test rod.

Table 2 - Tally score for the three channels for Al test rod loaded into the experimental cell

\begin{tabular}{|l|l|}
\hline & Flux (a.u.) \\
\hline Neutron Flux for right channel $c_{r}$ & $3.7310^{-08} \pm 1.4 \%$ \\
\hline Neutron Flux for center channel $c_{c}$ & $3.7110^{-08} \pm 1.4 \%$ \\
\hline Neutron Flux for left channel $c_{1}$ & $3.7210^{-08} \pm 1.4 \%$ \\
\hline
\end{tabular}

$$
\mathrm{s} / \mathrm{N}_{\mathrm{ALU}}=\mathbf{- 0 . 3 \%} \pm \mathbf{1 . 7 \%}
$$

The SNR is contained in the interval $[-2.0 \% ; 1.5 \%]$. As shown from this results, in the case of the aluminum test rod, the computed SNR is close to 0 , as expected.

This was considered as promising results for this preliminary modelling, and further analysis has to be realized with irradiated fuel pins, in order to increase the validation database of the model.

\section{Scattering coefficient}

It is also possible to evaluate the scattering coefficient $\alpha$, representing the effect of water loop structures on neutrons coming from the test rod, with the methodology described in section III.B. In Table 3 results obtained with the two options for the fresh $\mathrm{UO}_{2}$ fuel are presented.

Table 3 - Tally score for the central channel using different elastic scattering contribution evaluation options

\begin{tabular}{|c|c|c|}
\hline \multirow{3}{*}{$\begin{array}{c}S \\
(\mathrm{TAG} 1)\end{array}$} & Direct contribution & $9.6310^{-09} \pm 2.8 \%$ \\
\hline & $\begin{array}{l}(\mathrm{n}, \mathrm{xn}) \text { and }(\mathrm{n}, \mathrm{n} \text { ') reactions } \\
\text { on the test rod contribution }\end{array}$ & $2.7410^{-10} \pm 15.0 \%$ \\
\hline & $\begin{array}{l}\text { Elastic scattering } \\
\text { contribution into the test rod }\end{array}$ & $2.0210^{-09} \pm 17.0 \%$ \\
\hline \multirow{2}{*}{$\begin{array}{l}s+\alpha s \\
(T A G 3)\end{array}$} & Direct contribution & $1.5210^{-08} \pm 2.2 \%$ \\
\hline & $\begin{array}{l}(n, x n) \text { and }\left(n, n^{\prime}\right) \text { reactions } \\
\text { on the test rod contribution }\end{array}$ & $4.5810^{-10} \pm 11.6 \%$ \\
\hline
\end{tabular}

$$
\alpha=31.6 \% \pm 3.3 \%
$$

Therefore the scattering coefficient is contained in the interval [29.5\%;33.6\%] with this method. This value is consistent to the one find experimentally for the previously sodium loop implemented in CABRI, which was equal to $24 \%$. More scattering effect can be expected in the water loop than in sodium loop. As it is not foreseen to measure the scattering coefficient in the CABRI water loop, this new methodology will be applied in a next step to the previous sodium configuration in order to analyse the discrepancy to experimental data.

\section{CONCLUSION}

In conclusion, this work presents results obtained with a newly implemented, simplified, 2D model of the CABRI reactor. The purpose was to determine the "Signal to Noise Ratio" as well as the scattering coefficient, both involved in the treatment of the hodoscope measurements.

Thanks to this work, it is possible to predict with a good accuracy the SNR measured for the different pins soon tested in the framework on the CIP program. This new model can also play a tremendous role to control any modification linked to the test loop towards the hodoscope measurements, particularly in the phase of conception of new experimental programs.

A particular skill of the hodoscope is also to perform a quantitative analysis thanks to a fuel signal-to-mass conversion model for the fuel ejection into the coolant channel at pin failure. Histograms of masses that are built require the knowledge of the scattering coefficient [6]. For the previous experiments (sodium loop configuration), this parameter has been determined thanks to a specific experimental campaign. For the Pressurized Water Loop configuration, the parameter introduced in the analysis will be the calculated one. Hence this work shows the possibility to access to such kind of information with acceptable uncertainties and reasonable calculation time.

Results presented in Table 4 show the quite good consistency between these preliminary simulated values and the measured ones. A more complete analysis of the discrepancy between measures and calculation has now to be done, by performing sensitivity studies and increasing the experimental validation database.

Table 4 - Synthesis of the results

\begin{tabular}{clll}
\hline & \multicolumn{2}{c}{ Simulation } & \multicolumn{2}{l}{ Measurement } \\
s/N & Fresh UO & $28.3 \% \pm 1.7 \%$ & $27.0 \% \pm 2.0 \%$ \\
\cline { 2 - 4 } & Aluminum & $-0.3 \% \pm 1.7 \%$ & NA \\
\hline \multirow{2}{*}{$\alpha$} & $31.6 \% \pm 3.3 \%$ & $\begin{array}{l}23 \% \\
\text { loop conditions) }\end{array}$ \\
\hline
\end{tabular}

\section{NOMENCLATURE}

Signal of neutrons coming from the test rod Scattered neutrons coming from the test rod but useless for fuel displacement characterization The scatter coefficient, $s_{c}:=\alpha s$

Noise, neutrons coming for the CABRI reactor $n+s_{c}$

$c_{i} \quad \begin{aligned} & \text { Counting rate of the } \mathrm{i}^{\text {th }} \text { column (r=right, } \mathrm{c}=\text { center, } \\ & \text { and } \mathrm{l}=\mathrm{left})\end{aligned}$

$c_{i} \quad \begin{aligned} & \text { Counting rate of the } \mathrm{i}^{\text {th }} \text { column ( } \mathrm{r}=\mathrm{right}, \mathrm{c}=\text { center, } \\ & \text { and } \mathrm{l}=\mathrm{left} \text { ) }\end{aligned}$

$b_{j_{i, T A G} \text { option }}$ The value into bin $\mathrm{j}^{\text {th }}$ :

$\begin{array}{ll}- & 1=\text { test rod signal, } \\ \text { - } & 2=\text { test rod }(\mathrm{n}, \mathrm{xn}) \text { or }\left(\mathrm{n}, \mathrm{n}^{\prime}\right) \\ \text { - } & 3=\text { elastic scattered neutrons, } \\ \text { - } & 4=\text { all other contributions }\end{array}$

of the $i^{\text {th }}$ column ( $r=$ right, $c=c e n t e r$, and $\left.l=l e f t\right)$ evaluated with TAG option (TAG1 or TAG3) 


\section{REFERENCES}

[1] "Reactivity Initiated Accident transient testing on irradiated fuel rods in PWR conditions: The CABRI International Program". Biard B., et al. Annals of Nuclear Energy, Vol 141, 15 June 2020, 107253. https://doi.org/10.1016/j.anucene.2019.107253

[2] "Renovation, improvement and experimental validation of the Helium-3 transient rods system for the reactivity injection in the CABRI reactor". Duc B. et al. Proc of IGORR 2014 conference, Bariloche, Argentina, November 2014

[3] "The CABRI fast neutron Hodoscope: Renovation, qualification program and first results following the experimental reactor restart". Chevalier V., Mirotta S., Guillot J., Biard B. EPJ Web of Conferences, Volume 170, 10 January 2018

[4] “MCNP® user's manual. Code version 6.2. Manual Rev. 0" Werner C. J. editor. LA-UR-17-29981. October 27, 2017

[5] "The CABRI fast neutron Hodoscope: calibration campaign results". Chevalier V., Mirotta S., Monchalin N., Guillot J. The European Research Reactor Conference, the annual gathering of the research reactor community in Europe, RRFM, Oct 2020, HELSINKI (on line), Finland. (https://hal.archives-ouvertes.fr/hal-03080772).

[6] "Quantitative fuel motion determination with the CABRI fast neutron hodoscope: Evaluation methods and results." Baumung K., Augier G., Nuclear Technology, vol 96, p. 302, June 1991 